\title{
Disruption Management in Passenger Railway Transportation
}

Julie Jespersen-Groth ${ }^{\mathrm{a}, \mathrm{b}}$, Daniel Potthoff ${ }^{\mathrm{c}, \mathrm{e}}$, Jens Clausen ${ }^{\mathrm{a}, \mathrm{b}}$, Dennis Huisman $^{\text {cee,f }}$, Leo Kroon ${ }^{\text {d,e,f }}$, Gábor Maróti*d,e ${ }^{*}$ and Morten Nyhave Nielsen ${ }^{\mathrm{a}, \mathrm{b}}$

${ }^{a}$ DSB S-tog, Denmark

${ }^{\mathrm{b}}$ Department of Informatics and Mathematical Modelling Technical University of Denmark, DK-2800 Kongens Lyngby, Denmark

${ }^{c}$ Econometric Institute, Erasmus University Rotterdam P.O. Box 1738, NL-3000 DR Rotterdam, The Netherlands

${ }^{\mathrm{d}}$ Rotterdam School of Management, Erasmus University Rotterdam P.O. Box 1738, NL-3000 DR Rotterdam, The Netherlands

${ }^{\mathrm{e}}$ Erasmus Center for Optimization in Public Transport (ECOPT)

${ }^{\mathrm{f}}$ Department of Logistics, Netherlands Railways P.O. Box 2025, NL-3500 HA

Utrecht, The Netherlands

*This research was partially sponsored by the Future and Emerging Technologies Unit of EC (IST priority, 6th FP), under contract no. FP6-021235-2 (ARRIVAL). 


\section{Contents}

1 Introduction 3

2 Description of disruption management $\quad 7$

2.1 The capacity of a railway network . . . . . . . . . 8

2.2 Organizations . . . . . . . . . . . . . . . . 10

2.3 Actors . . . . . . . . . . . . . . . . 12

2.4 Processes . . . . . . . . . . . . . . . 13

2.5 Organizational issues . . . . . . . . . . . . 15

2.6 Related fields . . . . . . . . . . . . . . . . . . 16

3 Timetable adjustments $\quad 18$

3.1 Problem description . . . . . . . . . . . . . . . 18

3.2 Dispatching rules . . . . . . . . . . . . . . . . 20

3.2.1 Overtaking and changing stopping patterns . . . . 20

3.2 .2 Inserting an on-time train . . . . . . . . . . . 21

3.2.3 Increasing Residual Capacity . . . . . . . . . . . . . 21

3.3 Larger disruptions . . . . . . . . . . . . . . . . 23

3.4 A comparison with the airline industry . . . . . . . . . . 24

4 Rolling stock re-scheduling $\quad 25$

4.1 Problem description . . . . . . . . . . . . . . 25

4.2 Aircraft re-scheduling . . . . . . . . . . . . . . 27

4.3 Rolling stock re-scheduling at S-tog . . . . . . . . . . . . . . . . . . . . . . 39

4.4 Rolling stock re-scheduling at NS . . . . . . . . . . . 30

5 Crew re-scheduling $\quad 31$

5.1 Problem description . . . . . . . . . . . . . . 31

5.2 Crew re-scheduling at airlines . . . . . . . . . . . . 33

5.3 Current practice at S-tog . . . . . . . . . . . 34

5.4 Crew re-scheduling at NS . . . . . . . . . 36

$\begin{array}{lll}6 & \text { Integrated Recovery } & 37\end{array}$

$\begin{array}{lll}7 & \text { Conclusions } & 38\end{array}$ 


\begin{abstract}
This paper deals with disruption management in passenger railway transportation. In the disruption management process, many actors belonging to different organizations play a role. In this paper we therefore describe the process itself and the roles of the different actors.

Furthermore, we discuss the three main subproblems in railway disruption management: timetable adjustment, and rolling stock and crew re-scheduling. Next to a general description of these problems, we give an overview of the existing literature and we present some details of the specific situations at DSB S-tog and NS. These are the railway operators in the suburban area of Copenhagen, Denmark, and on the main railway lines in the Netherlands, respectively.

Since not much research has been carried out yet on Operations Research models for disruption management in the railway context, models and techniques that have been developed for related problems in the airline world are discussed as well.

Finally, we address the integration of the re-scheduling processes of the timetable, and the resources rolling stock and crew.
\end{abstract}

\title{
1 Introduction
}

Many Europeans travel frequently by train, either to commute or in their leisure time. Therefore, the operational performance of railway systems is often discussed in the public debate. Travelers expect to arrive at a specific time at their destination. If they travel by rail, they expect to arrive more or less at the time published in the timetable. However, unforeseen events often take place, which cause delays or even cancelations of trains. As a result, passengers arrive later than expected at their final destinations. Due to missed connections, the delay of a passenger can be even much larger than the delays of his individual trains.

Due to the importance for the public on one hand and the deregulation of the railway market on the other, railway operators now put more emphasis on their operational performance than in the past. Furthermore, due to the separation of the management of the infrastructure and the operations in 
many European countries (including Denmark and the Netherlands), several organizations are responsible for the performance of the railway system.

This paper deals with passenger railway transport only. However, in addition to the passenger railway operator itself, the infrastructure manager and other (also cargo) operators have a strong influence on the performance of the railway services of that single operator. Therefore, the role and the objectives of the infrastructure manager and of the operators are discussed.

We consider two passenger railway operators in more detail: DSB S-tog and NS. DSB S-tog is the operator of local train services in the greater Copenhagen area, see Figure 1. NS is the main operator in the Netherlands, having the exclusive right to operate passenger trains on the so-called Dutch Main Railway Network until 2015, see Figure 2. Both companies operate a set of lines on their network, where a line is defined as a route between two stations operated with a certain frequency, e.g. line A of S-tog runs between Hillerød and Hundige every 20 minutes.

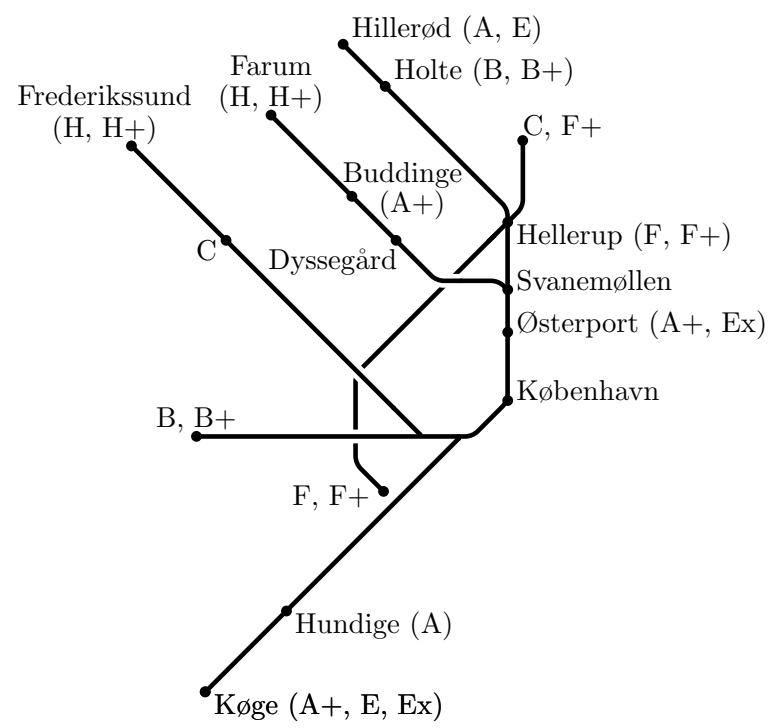

Figure 1: The S-tog railway network

Unfortunately, trains do not always run on time due to unexpected events. Examples are infrastructure malfunctions, rolling stock break downs, accidents, and weather conditions. Such events are called disruptions. To 


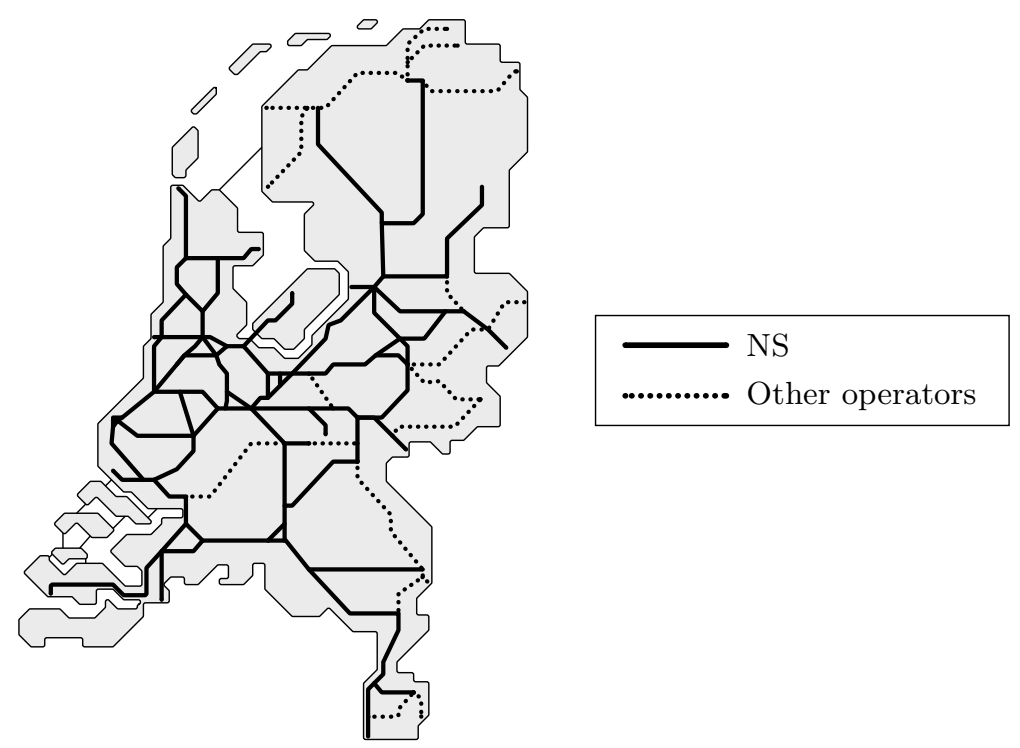

Figure 2: The Dutch railway network (in 2005)

give an indication, the numbers of disruptions related to infrastructure in the Netherlands during the first half of 2006 are reported in Table 1.

\begin{tabular}{l|rrr} 
Class & Disruptions & Avg. duration & Total duration \\
\hline Technical failure & 1656 & 2.2 & 3680 \\
Third parties & 1471 & 1.0 & 1491 \\
Weather & 172 & 2.3 & 393 \\
Others & 693 & 1.7 & 1208 \\
\hline Total & 3992 & 1.7 & 6772
\end{tabular}

Table 1: Disruptions in the Netherlands related to infrastructure during the first half of 2006 (ProRail [22])

Table 1 shows that the Dutch railway network has approximately 22 disruptions related to the infrastructure per day with an average duration of 1.7 hours. Note that disruptions caused by the operators, e.g. rolling stock break downs and crew no-shows are not reported in this table. The proportion between the disruptions caused by the operators and the infrastructure is roughly $50-50$ in the Netherlands. 
Different information is recorded for S-tog. Table 2 shows the number of affected trains in an average month for 2006. An affected train is either at least 2.5 minutes late on departure or canceled. Table 3 further details the information regarding that part of the affected trains where the disruption is contributed to S-tog.

\begin{tabular}{c|c|c|c} 
Responsible & Infrastructure manager & S-tog & Externally caused \\
\hline Affected trains & 4746 & 3981 & 660
\end{tabular}

Table 2: Disruptions in the S-tog traffic for an average month in 2006 subdivided according to responsability.

\begin{tabular}{c|c|c|c|c|c|c} 
Responsible & Rol. St. & Drivers & Dispatch. & Maint. & Pass. & Misc. \\
\hline Affected trains & 1131 & 665 & 88 & 44 & 1737 & 316
\end{tabular}

Table 3: Disruptions contributed to S-tog for an average month in 2006 (in total 3981) subdivided according to cause.

Of course, infrastructure managers and operators try to avoid disruptions. Unfortunately, many of them are hard to influence. Therefore, it is very important to limit the consequences of these disruptions. A very common problem in railways is that, due to the strong interdependencies in the railway network and due to cost efficient resource schedules, disruptions are very likely to spread over the network in space and time. This well-known phenomenon is called knock-on effect. The key to a good performance of railways is to limit the knock-on effect and thereby to limit the impact of single disruptions. Therefore, effective disruption management is required.

So far, Operations Research (OR) models have hardly been applied in practice for disruption management in railway systems. Nevertheless, it is our strong belief that OR models can play an important role to limit the impact of disruptions and thereby to improve the performance of railway systems. This belief is supported by the fact that nowadays OR models and techniques play a major role in several railway companies during the planning phase, where the focus is on a good balance of the service level 
offered to the passengers and efficiency of the resources rolling stock and crew. For an overview on these models and techniques, we refer to surveys of Assad [2], Cordeau et al. [5], and Huisman et al. [10]. Moreover OR models have proven to be quite effective already for supporting disruption management processes in the airline context, see e.g. Yu et al. [35].

The objectives of this paper are twofold. First, we intend to give a comprehensive description of the problems arising in disruption management for railway systems. Second, we aim at attracting new researchers to this field by describing the challenges that railway companies are faced with to improve their operational performance.

The remainder of this paper is organized as follows. In Section 2 we give a description of disruption management for railway systems, including a description of organizations and actors involved in this process. In Sections 3-5, we discuss timetabling, rolling stock and crew aspects of the disruption management process. Section 6 deals with the advantages and possibilities of integrating some of these processes. Finally, we finish the paper with some concluding remarks in Section 7.

\section{Description of disruption management}

Clausen et al. [4] give the following definition of a disruption in relation to airline operations: "An event or a series of events that renders the planned schedules for aircraft, crew, etc. infeasible." By definition, a disruption is hence a cause rather than a consequence. In this paper we use the same definition for railway operations, substituting "aircraft" with "rolling stock".

A disruption does not necessarily have immediate influence on the timetable - some disruptions like a track blockage renders the planned timetable immediately infeasible, while others as e.g. shortage of crew due to sickness may lead to cancelations either immediately, in the long run or not at all, depending on the amount of stand-by crew. Note that a disruption leads to a disrupted situation. Even though this is a slight abuse of terms, we will occasionally refer to the disrupted situation as the disruption itself.

Accordingly, we define railway disruption management as the joint approach of the involved organizations to deal with the impact of disruptions 
in order to ensure the best possible service for the passengers. This is done by modifying the timetable, and the rolling stock and crew schedules during and after the disruption. The involved organizations are the infrastructure manager and the operators.

Of course, one first has to answer the question if the situation is disrupted, i.e. if the deviation from the original plan is sufficiently large or not. Similar to the airline world (see Kohl et al. [13]), this question is normally answered by dispatchers monitoring the operations. In the railway world, however, it seems to be more difficult to judge an overall situation, even for experienced dispatchers. The latter is in particular true in case of a dense railway system. In the reminder of this paper, this issue is not considered further.

In Section 2.1 we define terms enabling us to describe and discuss capacity issues in railway networks. The Sections 2.2 to 2.4 introduces a framework of organizations, actors and processes in disruption management, which is valid for several European railway systems. In Section 2.5 we discuss the organizatorial context of the disruption management process and in Section 2.6, we describe a number of issues that are related to disruption management, such as robust planning.

\subsection{The capacity of a railway network}

The state of the daily operation of a train operator at some point in time is influenced by a number of factors, including the current state of the infrastructure (the rail network), and the state of all resources necessary in the operational phase, most notably rolling stock and crew. In the following we introduce the concepts of infrastructural capacity, operational capacity, utilization, and residual operational capacity.

The infrastructural capacity $I C(t)$ of a rail network $N$ in a particular state is the maximum amount of traffic which is continously able to flow through $\mathrm{N}$ in this state. The state may be described by the status of a number of parameters as e.g. the set of available tracks and for each track the state with respect to signals, and the maximum allowed speed for each track segment. Note that $I C(t)$ is independent of the current amount of 
traffic. The maximum value of $I C(t)$ over all possible states is sometimes referred to as the capacity of $N$.

At any point $t$ in time, the network $N$ and the resources are in one of their possible states. The operational capacity $O C(t)$ of the network is the maximum amount of traffic which is continously able to flow through $N$ with the current states for network and resources. Note that $O C(t)$ is always less than or equal to $I C(t)$ for $N$ in the current network state - one can never run more traffic than the infrastructural capacity allows for, but may not have resources enough to utilize this completely.

The utilization $U(t)$ of the network at time $t$ is the amount of continuously flowing traffic in the network $N$ at time $t$. Through the operational capacity of the network, $U(t)$ is depending on both the network state and the state of each resource. Note that a number of feasible values for utilization exist for each set of states for the network and resources. $U(t)$ is always less than or equal to $O C(t)$. The residual operational capacity or just the residual capacity at time $t$ is now the difference between the operational network capacity and the utilization at time $t: R(t)=O C(t)-U(t)$.

The states of the network and each of the resources are dynamic. The states are influenced by planned actions as inserting or taking out rush-hour trains, new crew meeting in, and trains taken out for maintenance. However, the states are also influenced by disruptions as e.g. engine break downs, inserting stand-by crew or rolling stock, or taking out train lines. A disruption typically decreases either the operational capacity, the utilization or both, while a recovery action typically increases either the residual capacity, the network utilization, or both. Since the utilization is less than or equal to the operational capacity, a decrease in operational capacity can never lead to an increased residual capacity.

Increasing the residual capacity may be achieved e.g. by decreasing utilization (e.g. canceling trains or entire train lines). Note that this operation does not necessarily increase the operational capacity. The state of the system may be changed to a state with larger operational capacity by e.g. allowing trains to run faster, decreasing the turn-around times at end stations, or inserting stand-by resources. This does not automatically increase the utilization. 
Finally note that a recovery action in general serves two distinct but often conflicting purposes: Increasing the network utilization, and changing the states of the resources to more preferable states. Canceling a train is very good from the resource perspective in that the action increases the residual capacity of the network as well as the available amount of both crew and rolling stock, and thereby possibly the operational capacity, but at the cost of a decreased utililization. Moreover, the "goodness" of a particular state may be difficult to quantify. For example, a state at time $t$ is usually considered good, if it is "close" to the planned state.

\subsection{Organizations}

The organizations directly involved in disruption management are the infrastructure manager and the railway operators. These organizations usually have contracts with the involved government. Moreover, they have a certain relationship with each other. These issues are described below.

The infrastructure manager has a contract with the government that obliges it to provide the railway operators with a railway network of a certain infrastructure capacity and reliability. The infrastructure manager has also the responsibility of maintaining the railway network as efficiently as possible.

A passenger railway operator obtains from the government a license to operate passenger trains on the network. The operator is contractually bound to provide a performance that exceeds certain specified thresholds on certain key performance indicators. For example, there may be thresholds for the number of train departures per station, for the (arrival) punctuality at certain stations, for the percentage of catched connections, for the seating probability, etc. Here, the punctuality is the percentage of trains arriving within for example 3 or 5 minutes of their scheduled arrival time at certain stations. The realization figures on these performance indicators have to be reported to the government periodically. If an operator does not reach one of the thresholds, it has to pay a certain penalty to the government. If the performance is very poor, another operator may be given the license to operate trains on the network. 
As a consequence, usually the main objective of the railway operator is to meet all thresholds set in the contract with the government at minimum cost. The latter is due to the fact that the railway operators are commercially operating companies. Thus the number of rolling stock units on each train must match with the expected number of passengers. Deadheading of rolling stock units between depots and to and from maintenance facilities must be minimized. Furthermore, the number of crews needed to run the operations and to cover unforeseen demand must be minimized as well.

In more detail, an important objective of the operators in the disruption management process is to minimize the number of passengers affected by the disruption, and to minimize the inconvenience for the affected passengers. Indeed, small delays of trains are usually not considered as a bad service by the passengers, but large disruptions are. If passengers are too often confronted with large disruptions, which usually lead to long extensions of travel times and, even worse, to a lot of uncertainty about travel options and travel times, they may decide to switch to a different mode of transport. In relation to this, passenger operators usually prefer to return to the original timetable as soon as possible after a disruption. Indeed, the original timetable is recognizable for the passengers. Therefore, the original timetable provides a better service than a temporary ad hoc timetable during a disruption.

The passengers are the direct customers of the railway operators, and they are only indirect customers of the infrastructure manager. This may imply that the manager has less knowledge of the expected passenger demand on each train and of the real-time passenger locations in the operations. The latter may prohibit a passenger focused dispatching, and may instead lead to a network capacity focused dispatching, i.e. dispatching focusing on supplying sufficient buffer times in the network to recover from disruptions.

Furthermore, each delay of a train may be attributed either to a railway operator or to the infrastructure manager, depending on the nature of the disruption. However, this creates a natural conflict between the organizations that may prohibit an effective communication and co-operation in the operations. The latter may be counter-productive for the operational performance of the railway system. Thus, although the infrastructure man- 


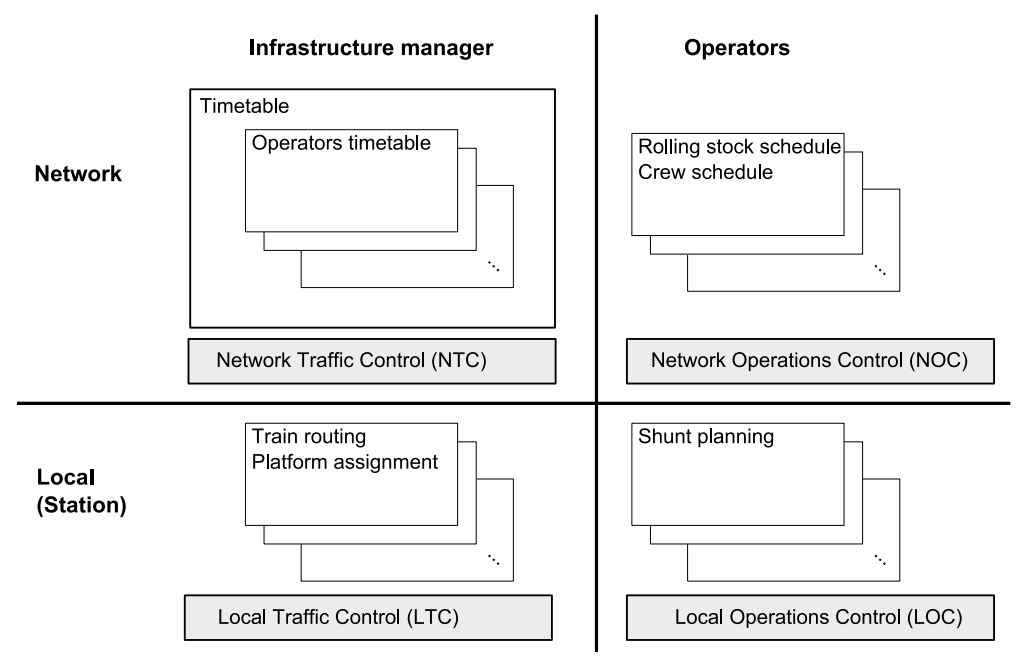

Figure 3: Schematic view of actors, timetables and resource schedules

ager and the railway operators have the same general objective of providing railway services to the passengers of a high quality level, there are also conflicting elements in their objectives.

\subsection{Actors}

In railway disruption management, the actors are the dispatcher of the infrastructure manager and those of the railway operators. The major tasks to be carried out are timetable adjustment, rolling stock re-scheduling, and crew re-scheduling. Figure 3 shows how the responsibilities for the different elements are shared among the actors.

The infrastructure manager controls and monitors all train movements in the railway network. Network Traffic Control (NTC) covers all tasks corresponding to the synchronization of the timetables of the different operators. NTC has to manage overtaking, re-routing, short turning, or canceling trains in order to prevent them from queueing up. The latter is a permanent threat at the basically one-dimensional railway infrastructure. Queueing up of trains immediately leads to extensions of travel times.

On a local level, the process is managed by the Local Traffic Control (LTC). For example, LTC is responsible for routing trains through railway 
stations and for platform assignments. Safety is ensured by headways and automatic track occupancy detection systems.

The Network Operations Control (NOC) of each passenger operator keeps track of the operations of the operator on a network level. The dispatchers of NOC are acting as decision makers for the operator in the disruption management process. Depending on the size of the operator, there is one or more dispatchers for rolling stock and crew, respectively. These dispatchers monitor and modify the rolling stock and crew movements. NOC dispatchers are the counterparts of the dispatchers of NTC.

Dispatchers of the Local Operations Control (LOC) of the railway operators are responsible for coordinating several local activities at the stations, such as shunting processes. They support NOC by evaluating whether changes to the rolling stock schedules can be implemented locally.

Train drivers and conductors are also important elements in the disruption management process. They are usually the first ones that are confronted with passengers that are affected by a disruption. If train drivers and conductors work on different lines, they may carry a delay from one line to another. In order to avoid this situation, the crew dispatchers may have to modify several duties. Besides making the decisions, the dispatchers also have to instruct and sometimes to convince the crew members to carry out the modifications, see Section 5 .

\subsection{Processes}

NTC dispatchers constantly monitor the operations and have to decide if an actual situation is a disruption or will lead to a disruption in the near future. When this is the case, they start the disruption management process. Within this process, the original timetable may need to be changed. This is done by carrying out a dispatching plan. Figure 4 displays the information flows between the different actors in this process.

First, NTC determines all trains that are affected by the disruption. NOC of the corresponding operators must then be informed about the disruption and its direct consequences. In the next step, the dispatchers have to find out to which extent it is still possible to run traffic on the involved 


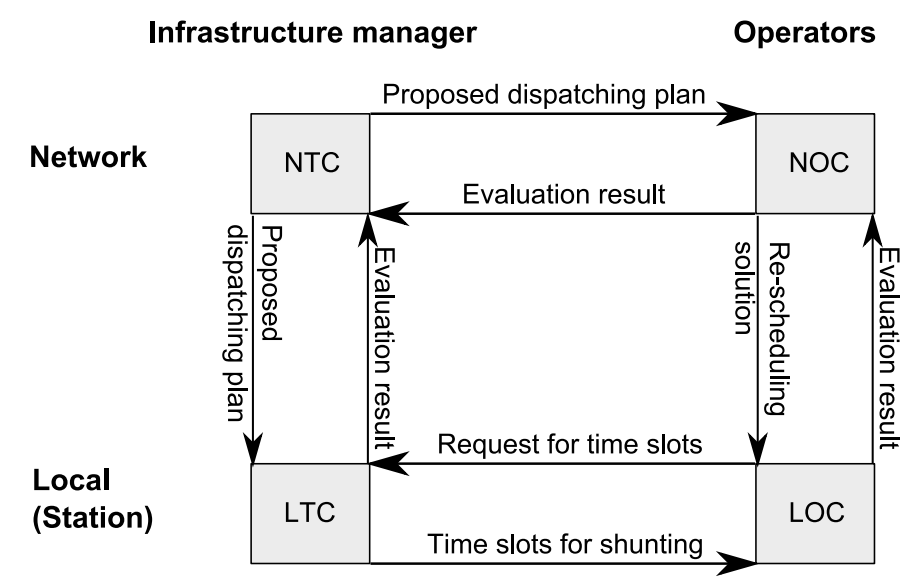

Figure 4: Information flow during the dispatching plan development

trajectory. Some pre-defined emergency scenarios give an indication about which trains should be overtaken, re-routed, short turned, or canceled. Using this information, an initial dispatching plan can be constructed. This dispatching plan must be evaluated by LTC. Almost simultaneously, the proposed dispatching plan is communicated to NOC of the operators. A complicating factor is the uncertainty about the duration of the disruption, for example NTC can only estimate how long it will take to repair a broken switch or signal.

The dispatching plan may correspond to changes in the planned operations of several operators. As a whole, these changes are compatible with respect to the safety regulations. However, for the operators it may be impossible to operate the dispatching plan due to their resource schedules for rolling stock or crew. Therefore, the decision about the dispatching plan is taken in consultation between the infrastructure manager and the operators.

Hence, NOC dispatchers have to check whether it is possible for them to operate the proposed dispatching plan. In particular, they have to check whether they can adapt their resource schedules to the proposed dispatching plan. Furthermore, LOC has to verify that the modified timetable and the adapted resource schedules can be carried out locally. Because of the combinatorial nature of the resource schedules and the limited time available, not all re-scheduling options can be evaluated. The re-scheduling solutions 
represent a trade-off between the available time and the quality of the solution.

This evaluation procedure can basically have three different outcomes. First, NOC and LOC may find a re-scheduling solution to the proposed dispatching plan where no additional cancelations or delays are needed. Second, they may find an initial solution, but trains have to be canceled in a second stage because rolling stock and/or crews are unavailable. A cancelation of a train has, however, a strong negative impact on the service level. Finally, NOC may come up with a request for changes to the proposed dispatching plan if this enables them to construct a much better solution.

Of course, not only one but several operators may ask for changes in the proposed dispatching plan. When these requests are conflicting, it is the responsibility of NTC to make a fair decision. This may involve another iteration of proposal and evaluation between NTC and the operators.

After the final decision about the dispatching plan has been taken by NTC, it is communicated to LTC and to the operators. LTC has to implement the new train routes and to change platform assignments. NOC has to inform the train drivers and conductors whose duties have been changed. LOC has to generate new shunting plans. LOC communicates directly with LTC to ask for time slots for shunting movements in the station area. Furthermore, passengers need to be informed in trains, at stations, and via internet and teletext about the changes in the timetable and alternative travel routes.

\subsection{Organizational issues}

The description in Section 2.3 of the actors in the disruption management process is a functional description, and not an organizational. For example, it suggests that all dispatchers of each of the mentioned actors are located in the same office. However, this need not be the case.

For example, in the Danish case, NTC, LTC and the timetable and rolling stock dispatcher of the NOC of S-tog are located in the same room, but the crew dispatcher of NOC is located at the crew depot of S-tog. This division was made on request of the train drivers. In practice, it creates some 
challenges regarding effective communication between the different dispatchers.

In the Netherlands, the situation is even more complex: the Netherlands have been split up into 4 regions, and each region has its own NTC office and its own NOC office of NS. Moreover, there is a central NOC office of NS for coordinating the rolling stock re-scheduling process. Similarly, there are 13 LTC offices and 13 LOC offices of NS. Obviously, this organizational split leads to a lot of additional communication within NTC and within NOC, which is counter-productive in the disruption management process. Therefore, there are currently plans to bring all offices of NTC together, and to do the same with the NOC offices. Moreover, it is investigated how the separation between the infrastructure manager and the operators can be reduced.

\subsection{Related fields}

Delay management is closely related to disruption management. Consider the following situation, typical for railway systems. For a passenger, even a small delay of a train can increase his travel time by 20,30 or 60 minutes if he misses a connection and has to wait for the next train. A similar situation exists for air traffic within a hub-and-spoke network when a flight arrives late at a hub. When the delay of a feeder train is not too large, it is possible to keep connections for passengers alive by delaying the departures of connecting trains a few minutes. The delay management problem is to find optimal wait-depart decisions for connecting trains such that the sum of the passenger delays is minimized. By keeping connections for passengers alive, an important criterion contributing to the service level of a railway system is addressed, namely the passenger satisfaction.

The wait-depart decisions correspond to minor changes to the original plans. The difference to disruption management is that, in delay management, it is usually assumed that the changes to the timetable can be conducted without re-scheduling rolling stock and crew, see Schöbel [25]. However, the decisions are taken by dispatchers of NTC and NOC that are also involved in disruption management, see Section 2.3. 
Another related issue is robust planning. Robust planning aims at making timetables and resource schedules less sensitive to disruptions. Robust planning approaches are called pro-active, since they take disruptions into account prior to their appearance.

There are two ways of interpreting robustness. The first one is to consider a plan robust if disruptions can be absorbed or the resulting knock-on effects can be reduced. We denote this property of a plan as the absorbing capacity. The second way of interpreting robustness is to consider a plan robust if it is well suited for recovery in case of disruptions. This property is called the recovery capacity of the plan.

The absorbing capacity of a plan is increased by introducing buffer times and by avoiding certain undesirable structures, such as short headways between trains, for which it is known that they are likely to propagate delays. Plans with a high absorbing capacity can compensate small disruptions completely, and they can reduce the consequences of larger ones. However, the high absorbing capacity usually comes at a price in terms of an increased cost of the planned operation.

Recovery capacity oriented robust planning is seeking plans that work well under one or several recovery strategies. Most recovery strategies use recovery actions that rely on certain desirable structures in the original plans. For example, initially planned crew connections can be swapped in the operations. In order to increase the recovery capacity of a plan, one tries to include such swapping options sufficiently and at the right locations in the plan. Moreover, plans are easier to recover when drivers and conductors stay together during their complete duty (the concept of train teams), and with the rolling stock. In a disrupted situation, adequate recovery strategies are easier to find when the recovery capacity of the plan is high.

Several methods have been proposed in order to increase the absorbing capacity of timetables. See Huisman and Boucherie [11], de Kort [6], Middelkoop and Bouwman [20], Soto y Koelemeijer et al. [29], and Kroon et al. [14] for recent developments in this area.

In order to create rolling stock circulations that less likely propagate delays, railway operators use planning rules based on experience. For example, the rolling stock circulations of NS are planned on a line-by-line basis and, 
preferably, each line is operated by a single rolling stock type, see Huisman et al. [10] and Fioole et al. [8]. No research has been done yet on more sophisticated methods for robust rolling stock planning.

Research on sophisticated methods for robust crew scheduling has so far only been done in the airline context. We refer to Ehrgott and Ryan [7], Schaefer et al. [24], and Yen and Birge [34] for methods to increase the absorbing capacity, and to Shebalov and Klabjan [26] for a method to increase the recovery capacity.

Stand-by rolling stock and crew planning are also interesting issues in the context of disruption management. During the planning phase, the number of stand-by rolling stock and crew and their positions have to be determined. To the best of our knowledge, this problem has not been addressed in the railway literature yet. A first reference dealing with a similar problem in the airline context is Sohoni et al. [27].

\section{Timetable adjustments}

\subsection{Problem description}

NTC has the overall responsibility of the railway operations and coordinates the disruption management process. When a disruption is recorded, NTC evaluates its effect and, if it is considered as severe, NTC tries to re-schedule the events of the timetable affected by the disruption.

The severeness of a disruption is not easily assessed. It is described as a combination of how much time will pass until the operations are according to plan again and how many trains will be affected. The number of passengers affected by a disruption also contributes to its degree of severeness. Finally, it makes a large difference to the severeness whether the headways between trains are small or large. For example, the effect caused by a blockage will be less on sections of the network with much time between the trains than on sections with little time between the trains.

Timetables are constructed with included buffer time. Therefore, a timetable is able to absorb some disruptions. Buffer times are included in the dwell times, the running times, and the headways. When a disruption oc- 
curs, the buffer times in the timetable are used to gain time whenever possible. Thus they enable recovery from a disruption.

The residual capacity of a railway network at a specific point in time is, as described in Section 2, a concept describing the capacity of the network in operation in relation to the traffic, i.e. how many trains are operated relative to the conditions of the network.

When a severe disruption occurs and it can not be absorbed by the buffers in the timetable, the utilization of the network decreases, and trains may queue up. In that case, NTC aims to increase the residual capacity in the network either by moving trains faster through the network, allowing overtaking at relevant stations, turning trains earlier, canceling departures, etc. Residual capacity is maintained by controlling the traffic flowing in the network and by preventing blocking situations to occur.

In Sections 3.2 and 3.3 we distinguish between disruptions with low and high impact on the timetable. Low level impact disruptions are those where recovery to the originally planned timetable is possible by using so-called dispatching rules. High level impact disruptions are those where recovery in this way is not possible, for example if a complete blockage occurs at some part of the network. In such a case, more significant recovery measures are needed.

A survey of optimization models for railway related problems is given by Cordeau et al. [5]. This survey describes various optimization models developed for railway problems. One of the described problems is the Train Dispatching Problem (TDP). TDP is the problem of minimizing delays by scheduling meets and overtakings, thereby taking into consideration operational costs. The velocity of trains is included in TDP as a decision variable.

Recently, a survey of algorithms and models for railway traffic scheduling and dispatching was given by Törnquist [32]. The problems mentioned are subdivided into tactical and operational scheduling and re-scheduling. Of specific interest is re-scheduling of trains, which focuses on the re-planning of an existing timetable when a disruption has taken place. 


\subsection{Dispatching rules}

Dispatching rules are used on disruptions that have a lower level of impact on the railway system. Dispatching rules are further divided into three subgroups according to the level of severeness of the disruption that invoked them. For disruptions with the lowest level of impact, where no substantial decrease in utilization has yet emerged, it is sufficient to make few modifications to the timetable. At the next level, where the traffic is more affected by the disruption, it is necessary to increase the utilization of the network. This can be done e.g. by increasing the operational capacity, for example through changes in the timetable in stopping patterns. The severest of the low impact disruptions need an increase in residual network capacity before recovery to a state with larger utilization (corresponding to the original timetable) is possible.

The different rules have different abilities to relieve disruptions and they have different effects for the passengers. From the passengers' point of view, a rule may affect the number of train departures per station or it may force the passengers to change their routes. The effect of a dispatching rule on the delays of trains and its effect on the passengers can be conflicting. Increasing the residual capacity often implies a decrease in the number of train departures, which is undesirable from the passengers' point of view. However, not increasing the residual capacity will make it very hard to absorb a delay, and this is also undesirable for the passengers.

\subsubsection{Overtaking and changing stopping patterns}

Handling operations is less complex if there is a predetermined order of train lines. In the case of a disruption, the predetermined order of lines can be broken on stations with multiple platforms in the same direction i.e. where overtaking between trains is possible. This is, for example, used when a fast train reaches a delayed stop train at a station with two platforms available in the same direction.

If a stop train is delayed and a fast train catches up with it, another possibility is to change their stopping patterns provided that the two trains are of the same rolling stock type and that it is impossible for the fast train 
to overtake the stop train. This rule is specifically used at S-tog. In practice, the passengers on the stop train are informed that after the next stop their train becomes a fast train. This enables them to get off in time if their destinations are stations where the fast train does not stop. The passengers on the fast train are informed similarly that their train becomes a stop train.

Note that in using both these rules no passenger experiences an additional delay on top of the initial delay caused by the disruption. If no action is taken in the latter situation, the fast train will queue up behind the stop train.

\subsubsection{Inserting an on-time train}

A dispatching rule, which is often used to prevent delays to spread over the network, is the insertion of an on-time train at an intermediate station. If a train is delayed at the first part of its route, it may be possible to insert a replacement train at an intermediate station on the route. The replacement train is inserted according to schedule. When the delayed train reaches the intermediate station, it is taken out of service. Seen from the passenger point of view, fewer departures are delayed. The rule has a limited effect on the overall delay. As no departures are canceled, no residual capacity is created.

\subsubsection{Increasing Residual Capacity}

Residual capacity is increased when departures are canceled. Canceling a departure from a terminal will increase the residual capacity along the entire route of the train. However, from the point of view of NOC, it leaves a train of some composition at the departure terminal. This might also force the cancelation of a departure at the terminal at the other end of the line. It may also create parking capacity problems at the shunting areas.

An alternative to canceling a departure completely is to skip stations along the route of a train, i.e. to change the stopping pattern of the train by decreasing the number of stops along its route. Stops canceled are mostly at stations with minor passenger loads and few connecting lines. 
Yet another alternative is to shorten the routes of trains. A train can be turned around before reaching its terminal, i.e. the remaining stations on its route are skipped, cf. Figure 5. Note that this is a dispatching rule for individual trains, in contrast to the emergency scenarios described in Section 3.3 where the routes of all trains of a line are shortened temporarily.

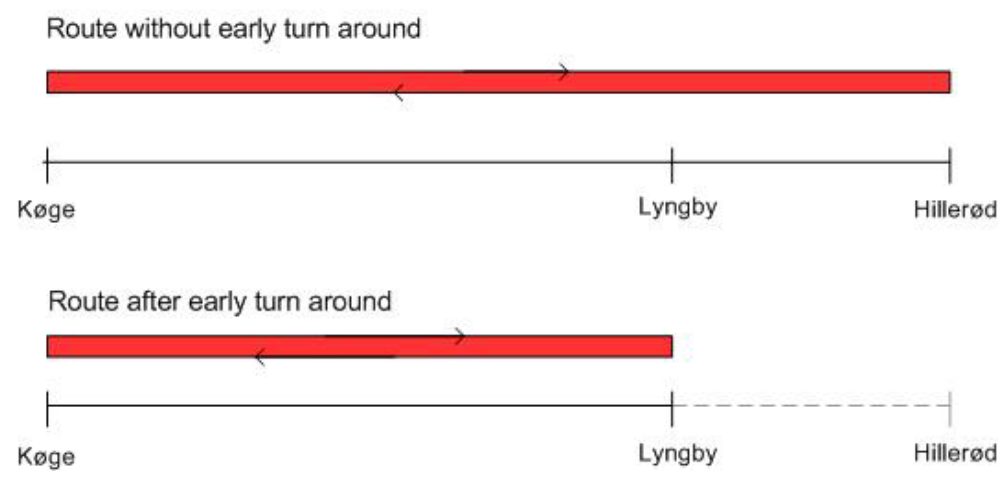

Figure 5: The train movement at early turn around

Finally, it is possible to cancel an entire train line. An example of how this dispatching rule is used in practice is the cancelation of line $\mathrm{B}+$, which is a line in the present S-tog timetable, cf. Figure 1. Suppose there is a delay in Hellerup. Due to signaling problems, the trains must run slower than indicated by the timetable. The lines operated on this route are lines $\mathrm{A}$ and E running from Hillerød and lines B and B+ running from Holte. To enable better absorbtion of the ongoing disruption, NTC decides to cancel line B+. The cancelation of line $\mathrm{B}+$ decreases the network utilization thereby allowing an increase in the headways between the remaining trains. In practice, the line is canceled by shunting trains on line $\mathrm{B}+$ to shunting areas as these are reached along the route of line $\mathrm{B}+$. Software for planning the later re-insertion of a canceled line is described in Section 4.3.

The advantages of the described dispatching rules are that they all increase the residual capacity for absorbing delays in the disrupted situation. The passengers, however, will experience that there are less departures, which may obstruct their travel plans. Also, if there was no time to couple extra train units to the trains still in operation, the seat capacity of these trains is most likely insufficient. Customer questionnaires show that, like 
delays and canceled departures, this is also considered as poor quality of service.

\subsection{Larger disruptions}

For high impact disruptions, a set of emergency scenarios may exist, e.g. when tracks in one or both directions are completely blocked. Usually, there is a separate plan for each section in the network and each direction.

The immediate reaction to a high impact disruption is to apply an appropriate emergency scenario. Usually, the headways are so tight that the system will queue up immediately if no adequate measures are taken after a high impact disruption has occurred. Therefore, usually all railway traffic is canceled around the disrupted area. Trains may be turned as closely as possible (according to their usual stopping pattern) to this location. Otherwise, trains may be rerouted, but this requires sufficient capacity on the detour route. Finally, some lines may be canceled completely.

As an example, consider a situation in which the tracks in both directions between stations Dyssegård and Buddinge near Copenhagen are blocked, see Figure 1. The lines crossing this section in a normal situation are the lines $\mathrm{A}+, \mathrm{H}$, and $\mathrm{H}+$. Line $\mathrm{A}+$ is operated between Køge and Buddinge, and lines $\mathrm{H}$ and $\mathrm{H}+$ are operated between Frederikssund and Farum. The emergency scenario for this blockage is presented in Tables 4 and 5 .

Table 4: Changes of the lines on the section Dyssegård to Buddinge

\begin{tabular}{|c|c|c|}
\hline Line & Changed from and to & Canceled from and to \\
\hline A+ & Køge to Østerport & Østerport to Buddinge \\
\hline H & $\begin{array}{c}\text { Frederikssund to Dyssegård } \\
\text { Buddinge to Farum }\end{array}$ & Dyssegård to Buddinge \\
\hline H+ & Frederikssund to Svanemøllen & Svanemøllen to Farum \\
\hline
\end{tabular}

Table 4 shows how the lines are changed and whether they are canceled partly or fully. Unless other disruptions occur, only the lines directly involved in the blockage are included in the emergency scenario. 
Table 5 specifies how many trains are necessary and which turnaround times must be used for them. Each line is changed according to its stopping pattern. Lines $\mathrm{A}+$ and $\mathrm{H}+$ are shortened, and therefore they can be run by 6 and 8 trains, respectively, whereas 8 and 10 trains are necessary normally. Line $\mathrm{H}$ is split into two parts and needs 8 plus 3 trains in the disrupted situation, whereas 10 trains are necessary normally.

Given the information in Tables 4 and 5, NTC knows which lines to cancel, where to launch bus-services, how many trains to use for each line, and how many train units to shunt to shunting areas.

Table 5: Turnaround times and necessary numbers of trains

\begin{tabular}{|c|l|l|}
\hline Line & Traffic south of blockage & Traffic north of blockage \\
\hline \hline A+ & $\begin{array}{l}\text { Køge to Østerport } \\
\text { Turnaround time: } 10 \text { min. } \\
\text { Trains necessary: } 6\end{array}$ & Canceled \\
\hline H & $\begin{array}{l}\text { Frederikssund-Dyssegård } \\
\text { Turnaround time: } 19 \text { min. } \\
\text { Trains necessary: } 8\end{array}$ & $\begin{array}{l}\text { Farum-Buddinge } \\
\text { Turnaround time: } 13 \text { min. } \\
\text { Trains necessary: } 3\end{array}$ \\
\hline H+ & $\begin{array}{l}\text { Frederikssund-Svanemøllen } \\
\text { Turnaround time: } 16 \text { min. } \\
\text { Trains necessary: } 8\end{array}$ & Canceled \\
\hline
\end{tabular}

\subsection{A comparison with the airline industry}

Due to the key differences in infrastructure of the underlying network, disruptions in the airline industry are handled differently than in the railway industry. The air transportation equivalent of NTC is Air Traffic Control, however, one cannot in general view ATC as an infrastructure manager. ATC is responsible for the air traffic with respect to safety both in the airports (airport control), and on the route of an aircraft (en-route control). Another difference is the number of operators sharing both airports and the airspace, which is usually much larger than what is experienced in the railway sector. 
Disruptions are in some sense much more serious for airlines than for railway companies, because the schedule contains much fewer connections between each origin and destination. Thus, a disruption usually has a much larger impact for the individual airline passengers than for railway passengers. Even then, the general pattern for dealing with a disruption in the airline sector is the same as in the railway sector: First solve the aircraft problem, then the crewing problem, then slots and gates, and then finally the passengers.

When an airline company experiences a disruption, the possibilities regarding timetable changes are very few: Either a departure can be delayed or it can be canceled. In the case of delay, the airline is in the same situation as a railway company: The aviation authorities have to assign a new slottime, and this requires free slots both in the relevants airports and on the route to be flown. Even though most traffic is routed through corridors in the airspace, the number of possible routes of an aircraft is not bound to a set of tracks layed out in 2 dimensions. From that point of view, the airline problem is much less complex than the corresponding railway problem.

Canceling an aircraft is always possible. However, this is considered to be the worst solution possible. The airlines are normally not bound to a contract specifying the service level and the amount of transportation to be delivered. Instead, competition among airlines servicing routes between the same destinations is a driving force in keeping the service level high.

\section{Rolling stock re-scheduling}

\subsection{Problem description}

This section describes rolling stock re-scheduling in a disrupted situation. Here the assumption is that, whenever this is necessary, the timetable has already been adjusted to the disrupted situation. The main goal is to decide how the rolling stock schedules can be adjusted to this new timetable at reasonable cost and with a minimum amount of passenger inconvenience.

The most characteristic feature of rolling stock is that it is bound to the tracks: rolling stock units cannot overtake one another, except at locations 
with parallel pairs of tracks. A broken rolling stock unit may entirely block the traffic - actually, this is a frequent cause of disruptions. Moreover, the operational rules of rolling stock units are largely determined by the shunting possibilities at the stations. Unfortunately, shunting is a challenging problem in itself, even for a medium-size station. Therefore, NOC must constantly keep contact with LOC and check whether or not their intended measures can be implemented in practice. The modifications may be impossible due to lack of shunting drivers or infrastructure capacity.

In case of a disruption, the first dispatching task is to keep the railway system running. These first decisions are taken under high time pressure. Timetable services must be provided with rolling stock of any type. Also, the assignment must fulfill some elementary requirements. For example, the rolling stock type must be compatible with the assigned trajectory, and each train should not be longer than the shortest platform on its route. Especially in a disrupted situation, shunting operations are reduced as much as possible. In particular, shunting operations at locations or points in time where they do not occur in the original schedules are highly undesirable.

Railway operators usually keep a certain amount of rolling stock on stand-by. These units can be used only in case of disruptions. Moreover, many of the rolling stock units are idle between the peak hours, since the rolling stock capacity is usually too large for off-peak hours. If a disruption takes place during off-peak hours, these idle units can act as stand-by units.

As a consequence of the first applied measures, the rolling stock units will not finish their daily duties at the locations where they were planned prior to the disruption. This is not a problem if two units of the same type get switched: rolling stock units of the same type can usually take each other's duty for the rest of the day. More likely, however, the numbers of units per type ending up in the evening at a station differ from the numbers of units per type that were planned to end up there. Thus, unless expensive deadheading trips are used, the traffic on the next day is influenced by the disruption. Modifications of the schedules for the busy peak hours of the next morning are highly undesirable. Therefore additional measures are to be taken so that the rolling stock balance at night is as close to the planned balance as possible. This problem is studied by Maróti [16]. 
Like disruption management in general, rolling stock re-scheduling has a stochastic character. For example, it can often only be estimated how long it will take to re-open certain temporarily unavailable infrastructure. Also, additional delays are likely to occur in a disrupted situation. Therefore, the dispatchers at NOC and LOC focus on the immediately forthcoming time period only, since planning for a longer period of time may be a waste of effort. They identify possible conflicts, and handle them in order of urgency.

After a disruption, it is preferable for the rolling stock schedules to return to the originally planned schedules as quickly as possible, since the feasibility of the originally planned schedules has been checked in detail.

A further important element in rolling stock re-scheduling is maintenance of rolling stock. Train units need preventive maintenance after a certain number of kilometers or days, roughly once a month. Due to efficiency reasons, units are usually in service just until they reach a certain maintenance limit. Units that are close to this limit and have to undergo a maintenance check in the forthcoming couple of days are monitored permanently. The latter is particularly important during and after a disruption which may have distracted the units from their planned route towards a maintenance facility. NOC has to make sure that these units reach a maintenance facility in time. Usually, only a small number of rolling stock units is involved in planned maintenance routings. Other units of a given type are interchangeable, both in the planning and in the operations.

The airline industry has similar processes when considering the shorthaul part of their operation, however, there are substantial differences for the longhaul part as described in the succeeding section.

\subsection{Aircraft re-scheduling}

The overall goal in airline disruption management is similar to the goal in railway disruption management: to get back to the optimized schedules with causing as little inconvenience for the passengers as possible.

A main difference between airline and railway systems is that trains usually consist of several rolling stock units. Moreover, the order of the 
rolling stock units in the trains may be relevant. Rolling stock units therefore interact in a more complex way with each other than aircraft do.

Moreover, pilots usually have a license for only one or two aircraft types, so swapping aircraft types inevitably leads to large-scale modifications of the crew schedules. As a consequence, the previously assigned aircraft type is changed in re-scheduling only if this is unavoidable. In order to reduce this problem, modern aircraft types may be split into families that can be flown by a single license. If each crew member has a license for just one type, the problem decomposes into subproblems for each fleet type. In a railway context, lack of knowledge about the rolling stock type is much less binding, since most train drivers have licenses for several rolling stock types. Thus rolling stock dispatchers have more freedom to modify rolling stock types.

Another important difference between airline and railway systems is the maintenance strategy. In the airline industry, each aircraft must undergo a larger safety check every 3 to 4 days - this can take place only at a small number of hubs. Therefore maintenance is often taken into account already in early planning phases when creating rotations for individual aircraft.

The term "tail numbering" or "tail assignment" is used for the process of assigning specific aircraft to specific departures. For shorthaul operations this happens ony a few days before the day of operation. Therefore, the rotations of aircraft are constructed to be maintenance feasible, i.e. to allow for maintenance checks within the intervals required by the aviation authorities.

For longhaul operations, the maintenance checks are also included in the rotations, but tail numbering takes place earlier than in the shorthaul case. In general, the rotations are planned to allow for some irregularities while maintaining maintenance feasibility.

Railway networks may contain many interconnected train lines. Most rolling stock units serve in a dozen of timetable services every day. This provides more exchange and correction possibilities for rolling stock units than what is usual in airline cases. A decision on aircraft routing can easily be irrevocable for many hours and in case of a longhaul operation even for a few days. 
Finally, from a revenue point of view, cancelation of a train is much less costly than that of a flight.

In the past years, substantial research has been done on aircraft rescheduling. Kohl et al. [13] and Clausen et al. [4] give excellent overviews.

\subsection{Rolling stock re-scheduling at S-tog}

In the case of a disruption affecting the rolling stock schedules, NOC reallocates rolling stock units to the train tasks. First of all, they aim to cover all tasks sufficiently with respect to the number of seats. There might not be enough time for shunting in each specific case i.e. allocating the right number of train units to a train is not possible. In this case, a train with a seat shortage is preferred over a canceled train.

At some rolling stock depots, space is an issue. Therefore, there can be some difficulties in finding a feasible rolling stock re-allocation.

Positioning data is not automatically supplied to the Rolling Stock Control System (RSCS) at S-tog. The data in the RSCS must therefore be updated manually by NOC during the operations. The updating of data is used respectively for reporting and statistics, and for giving information on the train lengths in real-time to the passengers. Having this information, the passengers will be able to locate themselves correctly on the platforms. As no automatic decision support or optimization system is available, the first feasible solution found is the one implemented in operation.

As mentioned in Section 3.3, a recovery method employed for large disruptions is canceling train lines. NOC at S-tog has the responsibility of determining a plan for the re-insertion of the train lines after the disruption. A model has been constructed for finding an optimized re-insertion plan, see Jespersen Groth and Clausen [12]. Based on the given number of trains that must be re-inserted from each depot along the line and the start time of the re-insertion, the model calculates which trains must be reinserted from which depots, and how the drivers for these trains can get to these depots. The automatic decision support system for re-inserting train lines is used in the operations. Moreover, in an ongoing project, the problem of re-allocating rolling stock units to trains in the operations is addressed. 


\subsection{Rolling stock re-scheduling at NS}

A remarkable property of the Dutch railway system is its density. This basically allows for many alternative rolling stock schedules through exchanges of train units. However, usually trains have short turn-around times, which rules out complex shunting operations at end points. Also, the shunting capacity (shunting area and crews) of stations is often a bottleneck.

Another complicating factor is that NS operates rolling stock of different types. Moreover, a train may contain units of different types. In this case, the order of the train units in the train is important. On one hand, this allows adjusting the rolling stock types well to the passenger demand. In case of disruptions, however, the dispatchers have the additional task of monitoring and re-balancing exchanged rolling stock types.

NOC and LOC of NS use an information system for monitoring and adjusting the rolling stock schedules. Tracking and tracing of train units provides information on the real-time locations of individual units. Moreover, the system matches the train units as well as possible with the duties in the actual version of the schedule. Since returning to the original schedule is important, the system represents the actual rolling stock schedule in terms of deviations from the original schedule.

The system does not include optimization modules, it only gives a warning if the rolling stock schedule has time or location conflicts. A new generation decision support system is currently being developed featuring an improved user interface and the possibility to incorporate optimization tools. These optimization tools are developed as part of on-going research at NS. The applicability of the models proposed by Fioole et al. [8] and Maróti [16] in the real-time operations will be further explored.

In the Netherlands, maintenance checks on rolling stock units can be carried out only at a few maintenance facilities. Therefore units routed for maintenance are paid special attention in the operations. Maróti and Kroon $[17,18]$ describe two integer programming models for maintenance routing. They take a rolling stock schedule of a few days as input and modify it so that the units that require maintenance soon can reach a maintenance 


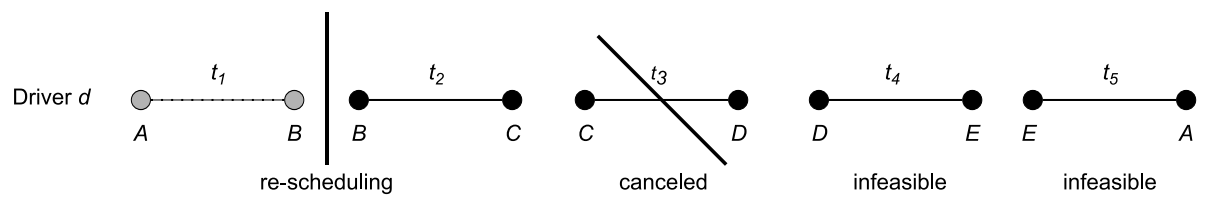

Figure 6: An infeasible duty

facility in time. The complexity of the problem is analyzed and a heuristic solution approach is suggested and tested on data of NS.

\section{Crew re-scheduling}

\subsection{Problem description}

Recall that the recovery of the timetable, the rolling stock schedule, and the crew schedule is usually done in a sequential fashion. For an estimated duration of the disruption, a modified rolling stock schedule has been determined for a modified timetable. Both are input for the crew re-scheduling problem, in which the crew schedule needs to be modified in order to have a driver and an appropriate number of conductors for each task of the modified timetable. Tasks can be either passenger train movements, empty train movements, or shunting activities.

The modified timetable contains the unchanged tasks from the original timetable which have not yet started and additional tasks which were created as reaction to the disrupted situation. For re-scheduling, the set of tasks of the modified timetable can be split into two subsets. The first subset contains all closed tasks, which are all tasks that are unchanged, not yet carried out, and part of an original duty which is still feasible. The second subset contains the open tasks, which include all additional tasks and all unchanged tasks that are assigned to an original duty which has become infeasible. A duty becomes infeasible due to a time or a location conflict. The latter may occur, e.g. when one of its tasks has been canceled, and hence the corresponding driver cannot execute the remaining part of his duty.

In Figure 6, we show an example of an infeasible duty. Because of a disruption, the train containing task $t_{3}$ is canceled. Driver $d$ has already finished task $t_{1}$ and is at station $B$. He can perform the next task in his 
duty, but since $t_{3}$ is canceled he cannot go from station $C$ to $D$. Hence, he will not be able to perform the two last tasks of his duty. Furthermore, this means that, if no action is taken, these two tasks need to be canceled as well. Moreover, driver $d$ has to get back to his crew depot at station $A$ in an appropriate way and at a reasonable time.

In order to prevent additional cancelations due to infeasible duties, the crew re-scheduling problem seeks to assign all open tasks to a crew member. A first possibility that can be used is re-assigning an open task to a crew member of another infeasible duty. Furthermore, an open task may be assigned to a stand-by crew located at a major station.

Since the amount of stand-by crew is limited, a set of feasible duties can also be taken into account for re-scheduling. These duties are broken up and their tasks are added to the set of open tasks. How to determine the set of duties to be broken up is an interesting problem itself. On one hand, the set must be small enough so that the resulting crew re-scheduling problem can be solved quickly, while on the other hand a too small set may not provide enough possibilities to cover the open tasks.

The possibilities for changing duties on the day of operation are based on rules and agreements between the railway company and labor unions. These possibilities usually vary from company to company. For example, the driver's route knowledge has to be taken into account as well as his license for certain rolling stock types. In order to increase the flexibility of the crews, they can be repositioned to another station by traveling on trains as passengers. This option is called crew deadheading.

The objective of the crew re-scheduling problem is a combination of different aspects, namely feasibility, operational costs, and stability. The feasibility aspect is by far the most important, since decisions need to be taken fast in a disrupted situation. It is the decision of the operator how to balance the aspects operational costs and stability.

First of all, there is the feasibility aspect. It is not evident that all open tasks can be covered by a solution. Given two solutions with different uncovered tasks, there may exist a preference for one of them, depending on the urgency and the expected numbers of passengers of the uncovered tasks. If a task cannot be covered, canceling it will lead to a feasible crew 
re-scheduling solution. An additional cancelation, however, leads to more inconvenience for the passengers, which is against the general aim of disruption management. Moreover, such a cancelation has to be approved by the rolling stock dispatchers and the local planners, since it disturbs the rolling stock circulation. Because a cancelation is a change of the timetable, it has to be approved by NTC.

Operational costs are the second aspect in the objective. In the railway context, the crew payments are often based on fixed salaries. Nevertheless, some parts of a re-scheduling solution influence the operational costs. Crew deadheading on trains can be considered to have no costs other than time, whereas using other transport options for repositioning and taking home stranded crews is not free. Also, operator specific compensations for extra work due to modified duties need to be considered.

The third aspect in the objective is stability. Humans are involved in the implementation of every re-scheduling solution and can cause its failure. A crew dispatcher may, for example, forget to call a driver and inform him about the modifications in his duty. Therefore, a solution is considered to be more stable if the number of modified duties is smaller.

To the best of our knowledge, only the paper of Walker et al. [33] deals with re-scheduling of train crews during disruptions. The paper presents a model that manipulates the timetable and the crew schedule at the same time. The objective is to simultaneously minimize the deviation of the new timetable from the original one, and the cost of the crew schedule. One part of the model represents the timetable adjustment, the other part corresponds to a set partitioning model for the crew schedules. Both parts are linked in order to get a compatible solution. It should be mentioned that the railway systems addressed in the research is of a relatively simple structure.

\subsection{Crew re-scheduling at airlines}

Crew re-scheduling has much more effect on the operational cost of an airline operator than of a railway operator. Because of its managerial relevance, airline crew re-scheduling on the day of operation has also become of growing interest for the research community during the last decade. 
Yu et al. [35] reports the savings that Continental Airlines has realized in three major disruptions due to the re-organization of their disruption management process and the installation of decision support systems. The used crew re-scheduling model is based on the prototype described by Song et al. [28]. A set covering model is formulated, based on a time-space network that represents possible modifications of crew pairings for a certain recovery period. Here a pairing is a sequence of flight legs and overnight rests that begins and ends at the same crew base, and that is to be carried out by a single crew member. This model is solved by depth-first Branch-and-Bound, where open flight legs are covered according to their urgency.

One structural difference between airline and railway crew re-scheduling is the time horizon. Due to more complex regulations for pilots, the position of a pairing within the roster has to be taken into account during re-scheduling (see Medard and Sawhney [19]). Extending a pairing over the planned duration can be infeasible due to roster regulations, such as a maximum working time per month. In the railway context, such rules can usually not be violated during re-scheduling. Therefore, usually only duty related rules have to be taken into account for railway crew re-scheduling.

Many approaches in the literature, like Stoiković et al. [30], Nissen and Haase [21], Medard and Sawhney [19], and Lettovský et al. [15], use column generation to solve set covering or set partitioning models for crew re-scheduling. The first three approaches use network formulations for the subproblems, whereas the last one uses an enumerative pairing generator. We refer to Clausen et al. [4] for a more detailed description of approaches to airline crew re-scheduling.

\subsection{Current practice at S-tog}

At DSB S-tog a year plan can be changed up to 72 hours before the day of operation, for instance due to work on tracks. Such a plan is called a special plan. A very strict restriction in a special plan is the start and end times which can only be moved up to 20 minutes earlier (resp. later). Within the last 72 hours before operations the content of the duties can still be changed without notifying the driver, but the start time cannot be moved earlier and 
the end time cannot be moved later. If such a move is needed, the planners at NOC must negotiate with the driver.

From 2006 a graphical dispatching system has been used to support the planners. For instance, the drivers have a sign-on terminal and the dispatcher has a real time picture of the drivers meant to sign on during the next half hour. Currently, the system does not contain decision support, which means that all operations are performed manually by planners. The system is currently being extended so that real time information of the train positions are fed to the system. Clearly, without such functionality, it is a tedious process to update the system in major disruptions.

The optimization software, TURNI, described e.g. in Abbink et al. [1], has been used for generating the annual standard day plans with great success and significant savings during the last couple of years. TURNI is based on a set covering model and dynamic column generation.

Recently, a number of trials have been made to use TURNI also for special plans. The idea used has also been tested at NSR, but due to the smaller problem size at DSB S-tog it seems more likely that S-tog will be able to use TURNI for special planning.

Since the dispatching problem is very similar to the operational planning problem at S-tog, the standard version of TURNI also has been tested for dispatching. The idea is to plan within a window of for instance 2 hours and remove all duties outside the window. The preliminary test with the system shows that approximately 20 minutes is required for a useful solution to be found. Of course, 20 minutes is too much in a disrupted situation, but on the other hand it seems likely that the (exact) solution method is applicable if some time is spent on a more tailored system for dispatching than the standard TURNI system. A potential speedup is to reduce the set of rules from the standard system, since the rules used in dispatching are less restrictive than the rules used for year plans and special plans.

A decision support system for train driver dispatchers is currently under development as a part of a Ph.D.-project supported by S-tog. A solution method to the Train Driver Recovery Problem, described in Rezanova and Ryan [23], is based on rescheduling a small part of the train driver schedule affected by a disruption. The problem is formulated asa set par- 
titioning problem andposesses strong integer properties. The proposed solutionapproach is therefore anLP-based Branch \& Bound algorithm. The LPrelaxation of the problem is solved with a dynamic column and constraint generation algorithm.Pilot experiments are very promising, both with regards to the integrality property and to the efficiency of the method.

The main objective is to minimize the number of changed duties. The main reason is the resulting communication problem if a large number of duties are changed, since the communication has to be performed manually by the crew dispatcher. A second objective is a robust plan where robustness is defined as large buffer times before breaks within the recovered duties. The main focus in the project is cancelations of entire train series (lines) for a period of time which is commonly used during larger disruptions. This has a large effect on the plans, since many duties are traditionally involved and a p-trip (where the driver travels as passenger) can potentially be canceled making it impossible for the driver to perform his next task.

\subsection{Crew re-scheduling at NS}

The crew dispatchers at NOC of NS use an interactive software system. This provides them with information about the actually planned duties, and enables them to store their duty modifications in the system. The system informs them about delays of trains and about modifications in the timetable and rolling stock schedules. The system also indicates time and location conflicts in the duties. Recovery options, however, have to be found manually without algorithmic support. In the manual procedure, open tasks are covered one at a time in order of urgency.

Several agreements exist about the way duties may be modified on the day of operation. For example, if a duty is modified, it should not end more than 30 minutes after the end of the original duty. Experiments were carried out to inform crew members automatically via SMS about duty modifications. However, direct communication may be more effective if a dispatcher discovers an option outside the standard rules. Since this negotiation process takes time, the dispatchers often prefer to use stand-by crew to cover open tasks whenever stand-by crew are available. 
Recently, Huisman [9] developed an algorithm for crew re-scheduling in the case of planned track maintenance. The algorithm is based on a combination of column generation and Lagrangian relaxation for solving a set covering type of model. A similar model is used by Nissen and Haase [21] for airline crew re-scheduling during disruptions. The difference is that, in the case of planned track maintenance, every original duty can be taken into account for re-scheduling, whereas in the latter approach only a subset of the duties is considered due to time limitations.

In an ongoing research project, it will be evaluated if the approach of Huisman [9] can be adapted to crew re-scheduling during disruptions. The first issue is how to choose the subset of original duties that should be broken-up and taken into account for re-scheduling. Furthermore, acceleration techniques for the column generation process like partial pricing and stabilization will be evaluated. Last but not least, heuristics that produce feasible solutions early in the column generation process may be of great benefit in the context of disruption management.

\section{$6 \quad$ Integrated Recovery}

In the airline industry the traditional sequence of recovery in case of a disruption is first to resolve the aircraft problem, then to crew this solution, handle the problems regarding infrastructure (gates, arrival/departure slots), and finally to take care of the rerouting of passengers.

This sequence has several drawbacks: Breaking the problem into subproblems may in itself lead to a suboptimal solution of the recovery problem since each subproblem has its own objective. As an example consider a disruption affecting a short roundtrip from a hub (e.g. Copenhagen - Stockholm - Copenhagen). From a resource point of view canceling the flight is the best reaction since no additional changes to aircraft and crew plans are necessary. However, from a passenger point of view this is the worst solution.

In the past there have been several attempts to construct integrated recovery systems. One approach has been to build dedicated recovery systems for aircraft, crew, and passengers, and then to combine these into an 
integrated tool. By iterating the recovery process between the dedicated systems this system then tries to find a solution, which from a holistic perspective is better than the individual solutions proposed by each dedicated system. Other architectures have been tried, for example building tools that in one system integrate the recovery of both aircraft and crew, cf. [31], and approaches taking into account passenger costs cf. [3].

Presently no system is capable of true integrated recovery. Due to the development in computational power and in the methods used in dedicated recovery systems, major software vendors as e.g. Jeppesen are, however, optimistic regarding the possibilies of building such system in the airline case.

The situation is quite diffent in the railway case. Major differences exist regarding the subproblems, which is apparent when one views the processes described in Section 2: In case of a disruption it is the NTC who in the end decides on the solution to be implemented. Furthermore, the possibilities for rerouting passengers are much better - it is often possible to increase the seat capacity of succeeding departures, while this is much more difficult when dealing with aircraft. The integrated recovery approach has therefore received little attention up till now. The benefits from such an approach compared to the sequential approach may, however, be large in terms of quality of service, and the field is expected to become an active research field in the future.

\section{Conclusions}

Railway operators pay much attention to improve their operational performance. One of the key issues is to limit the number of delays by reducing the knock-on effect of single disruptions. To achieve this goal, effective disruption management is required. In this paper, we have explained the role of the different organizations and actors in the disruption management process. An important issue here is that next to the operator itself, the infrastructure manager plays a major role in the disruption management process. The different objectives of both organizations on one hand and difficult commu- 
nication schemes on the other hand, complicates the disruption management process a lot.

After the description of disruption management, we have discussed the three subproblems arising in railway disruption management: timetable adjustment, and rolling stock and crew re-scheduling. To adjust the timetable, several different dispatching rules are applied in practice. Unfortunately, no optimization techniques are involved to solve this problem currently. For the re-scheduling of rolling stock and crew some first attempts have been made in the literature to come up with OR models and solution techniques. Most of these have been derived from similar problems in the airline world. However, most of these ideas are in an early stage and have not been applied in practice yet.

In other words, there is a major challenge for the OR community to develop new models and come up with new solution approaches to tackle these problems. Therefore, we hope and expect that another review paper on railway disruption management in about 5 years contains much more models and solution approaches than this one, and moreover that many of them have been applied in practice.

\section{References}

[1] E. J. Abbink, M. Fischetti, L. G. Kroon, G. Timmer, and M. J. C. M. Vromans. Reinventing Crew Scheduling at Netherlands Railways. Interfaces, 35(5):393-401, 2005.

[2] A. A. Assad. Models for rail transportation. Transportation Research A, 14:205-220, 1980.

[3] S. Bratu and C. Barnhart. Flight operations recovery: New approaches considering passenger recovery. Working paper, 2004.

[4] J. Clausen, A. Larsen, and J. Larsen. Disruption management in the airline industry - concepts, models and methods. Technical report, Informatics and Mathematical Modelling, Technical University of Denmark, DTU, Richard Petersens Plads, Building 321, DK-2800 Kgs. Lyngby, 2005. URL http://www2.imm.dtu.dk/pubdb/p.php?3763. 
[5] J.-F. Cordeau, P. Toth, and D. Vigo. A survey of optimization models for train routing and scheduling. Transportation Science, 32:380-404, 1998.

[6] A. F. de Kort. Advanced railway planning unsing Max-Plus algebra. In J. Allan, C. A. Brebbia, R. J. Hill, and G. Sciutto, editors, Computers in Railways VII, pages 257-266, Ashurst, UK, 2000. WIT Press.

[7] M. Ehrgott and D. M. Ryan. Constructing Robust Crew Schedules with Bicriteria Optimization. Journal of Multi-Criteria Decision Analysis, 11:139-150, 2002.

[8] P.-J. Fioole, L. G. Kroon, G. Maróti, and A. Schrijver. A rolling stock circulation model for combining and splitting of passenger trains. European Journal of Operational Research, 174:1281-1297, 2006.

[9] D. Huisman. A Column Generation Approach to solve the Crew Rescheduling Problem. to appear in European Journal of Operational Research, 2005.

[10] D. Huisman, L. G. Kroon, R. M. Lentink, and M. J. C. M. Vromans. Operations Research in passenger railway transportation. Statistica Neerlandica, 59(4):467-497, 2005.

[11] T. Huisman and R. J. Boucherie. Running times on railway sections with heterogeneous train traffic. Transportation Research Part B, 35: 271-292, 2001.

[12] J. Jespersen Groth and J. Clausen. Optimal reinsertion of cancelled train lines. Technical Report Report-2006-13, Informatics and Mathematical Modelling, Technical University of Denmark, August 2006.

[13] N. Kohl, A. Larsen, J. Larsen, A. Ross, and S. Tiourine. Airline disruption management - perspectives, experiences and outlook. Technical report, Informatics and Mathematical Modelling, Technical University of Denmark, DTU, Richard Petersens Plads, Building 321, DK-2800 Kgs. Lyngby, 2004. URL http://www2.imm.dtu.dk/pubdb/p.php?3311. 
[14] L. G. Kroon, R. Dekker, and M. J. C. M. Vromans. Cyclic Railway Timetabling: a Stochastic Optimization Approach. In F. Geraets, L. G. Kroon, D. Wagner, and C. Zaroliagis, editors, accepted for Algorithmic Methods for Railway Optimization, Lecture Notes in Computer Science, Berlin, 2005. Springer.

[15] L. Lettovský, E. L. Johnson, and G. L. Nemhauser. Airline Crew Recovery. Transportation Science, 34(4):337-348, 2000.

[16] G. Maróti. Operations Research Models for Railway Rolling Stock Planning. PhD thesis, Eindhoven University of Technology, Eindhoven, The Netherlands, 2006.

[17] G. Maróti and L. G. Kroon. Maintenance Routing for Train Units: the Interchange Model. Computers \&5 Operations Research, 34(4):11211140, 2007.

[18] G. Maróti and L. G. Kroon. Maintenance Routing for Train Units: the Transition Model. Transportation Science, 39(4):518-525, 2005.

[19] C. P. Medard and N. Sawhney. Airline crew scheduling from planning to operations. European Journal of Operational Research, 2006.

[20] D. Middelkoop and M. Bouwman. Train network simulator for support of network wide planning of infrastructure and timetables. In J. Allan, C. A. Brebbia, R. J. Hill, and G. Sciutto, editors, Computers in Railways VII, pages 267-276, Ashurst, UK, 2000. WIT Press.

[21] R. Nissen and K. Haase. Duty-period-based network model for crew rescheduling in European airlines. Journal of Scheduling, 9:255-278, 2006 .

[22] ProRail. Spoorcapaciteit eerste helft 2006 stabiel t.o.v. 2005 (track capacity first half 2006). Internet: http://www.prorail.nl/ProRail/Bedrijfsinformatie/Nieuws/Spoorcapaciteit+eerste+helft+2006+stabiel+t.o.v.+2005.htm, 2006. 
[23] N. J. Rezanova and D. M. Ryan. Solving the train driver recovery problem. Technical Report Report-2006-24, Informatics and Mathematical Modelling, Technical University of Denmark, December 2006.

[24] A. J. Schaefer, E. L. Johnson, A. J. Kleywegt, and G. L. Nemhauser. Airline Crew Scheduling Under Uncertainty. Transportation Science, 39(4):340-348, August 2005.

[25] A. Schöbel. Integer programming for solving the delay management problem. Technical Report 92, Technische Universität Kaiserslautern, Institut für Techno- und Wirtschaftsmathematik, January 2004.

[26] S. Shebalov and D. Klabjan. Robust Airline Crew Pairing: Move-up Crews. Transportation Science, 40(3):300-312, 2006.

[27] M. G. Sohoni, E. L. Johnson, and T. G. Bailey. Operational airline reserve crew planning. Journal of Scheduling, 9:203-221, 2006.

[28] M. Song, G. Wei, and G. Yu. A Decision Support Framework for Crew Management During Airline Irregular Operations. In G. Yu, editor, $O p$ erations Research in the Airline Industry. Kluwer Academic Publishers, Boston, 1998.

[29] G. Soto y Koelemeijer, A. R. Iounoussov, R. M. P. Goverde, and R. J. van Egmond. PETER, a performance evaluator for railway timetables. In J. Allan, C. A. Brebbia, R. J. Hill, and G. Sciutto, editors, Computers in Railways VII, pages 405-414, Ashurst, UK, 2000. WIT Press.

[30] M. Stoiković, F. Soumis, and J. Desrosiers. The Operational Airline Crew Scheduling Problem. Transportation Science, 32(3):232-245, August 1998.

[31] M. Stojković and F. Soumis. An Optimization Model for the Simultaneous Operational Flight and Pilot Scheduling Problem. Management Science, 47:1290-1305, 2001.

[32] J. Törnquist. Railway Traffic Disturbance Management. PhD thesis, Blekinge Institute of Technology, Karlskrona, Sweden, 2006. 
[33] C. G. Walker, J. N. Snowdon, and D. N. Ryan. Simultaneous disruption recovery of a train timetable and crew roster in real time. Computers \& Operations Research, 32:2077-2094, 2005.

[34] J. W. Yen and J. R. Birge. A Stochastic Programming Approach to the Airline Crew Scheduling Problem. Transportation Science, 40(6): 3-14, February 2006.

[35] G. Yu, M. Argüello, S. Gao, S. M. McCowan, and A. White. A New Era for Crew Recovery at Continental Airlines. Interfaces, 33(1):5-22, January-February 2003. 